\title{
Validação de modelos de sistemas econômicos baseada na tendência
}

\author{
Otávio Augusto Rodrigues de Oliveira Freitas * \\ Lucas Giovani Nardo* Vinicius da Silva Borges* \\ Samir Angelo Milani Martins* \\ Erivelton Geraldo Nepomuceno*

\begin{abstract}
* GCOM - Grupo de Controle e Modelagem, UFSJ - Universidade Federal de São João del-Rei - Pça. Frei Orlando, 170 - Centro 36307-352 - São João del-Rei, MG, Brasil (e-mail: otavio_augusto_freitas@hotmail.com; gnlucas@gmail.com; vnisb@hotmail.com; martins@ufsj.edu.br; nepomuceno@ufsj.edu.br
\end{abstract}

\begin{abstract}
System identification is an area of knowledge that it is present in many fields of science. One reason for the study of identification techniques is that it is relatively simple to analyze a model in comparison of the real system. This is valid for economic systems, since these systems are interesting for the study of identification techniques, as the financial market is extremely complex and volatile, making it difficult to model. This paper proposes a new validation index, the IAT (Trend Attain Index), since figuring out whether the behavior of a particular system will increase or decrease over time is as important as precisely indicating the next value at the next instant. Therefore, it can reduce the risks of a given investment. In order to study the application of the index, Brazilian inflation was modeled by the IPCA (Extended Consumer Price Index) through NARX polynomials. Finally, the obtained models were able to identify the trend month by month, presenting, in a given model, an $I A T=82.76 \%$.

Resumo: Identificação de sistemas é uma área do conhecimento que está presente em diversos campos da ciência. Uma das justificativas para o estudo das técnicas de identificação se dá pelo fato de ser relativamente simples a análise de um modelo em comparação com o sistema real. Tal afirmação é válida para sistemas econômicos, com acréscimo que esses sistemas são interessantes para o estudo de técnicas de identificação, pois o mercado financeiro é complexo e volátil, dificultando a sua modelagem. Dessa forma, este trabalho propõe um índice de validação, o IAT (Índice de Acerto de Tendência), uma vez que descobrir o comportamento de sistema, ao longo do tempo, é tão importante quanto indicar, precisamente, o próximo valor no instante seguinte, podendo então diminuir os riscos de um determinado investimento. Para estudar a aplicação do índice, modelou-se a inflação brasileira dado pelo IPCA (Índice de Preços ao Consumidor Amplo) via NARX polinomiais. Os modelos obtidos conseguiram identificar, portanto, a tendência mês a mês, chegando a apresentar, em um determinado modelo, um $I A T=82,76 \%$.
\end{abstract}

Keywords: System identification; NARX Models; Econometric Systems; Brazil Inflation Rate. Palavras-chaves: Identificação de Sistemas; Modelos NARX; Sistemas Econométricos; Inflação Brasileira.

\section{INTRODUÇÃO}

Modelos matemáticos de sistemas desempenham um papel essencial na ciência e tecnologia atualmente. As aplicações dos modelos vão desde simulação e previsão, até controle e diagnóstico em campos heterogêneos, como no ramo da engenharia (Waseem and Sadiq, 2018), economia (Nguyen, 2019), medicina (Buonomo et al., 2018) e muitos outros (De Nicolao, 1997).

Por isso, é natural estudar e aperfeiçoar as técnicas e os algoritmos que permitem construir modelos dinâmicos a partir de dados medidos. Dessa forma, para a cons-

\footnotetext{
* Este trabalho foi financiado pelos seguintes grupos de financiamento CNPq/INERGE, Fapemig e Capes.
}

trução de representações matemáticas, a tarefa clássica de identificação pode ser representada pelos cinco passos fundamentais: i) teste e coleta de dados, ii) escolha da representação matemática, iii) seleção da estrutura do modelo, iv) estimação de parâmetros e v) validação (Aguirre, 2004).

Desse modo, o setor financeiro é um campo interessante para o estudo de técnicas de identificação, uma vez que se trata de um sistema extremamente volátil. Outrossim, a criação de modelos confiáveis é necessária, visto que a previsão indicada no modelo pode, por exemplo, interferir na estratégia de mercado de várias equipes econômicas, tanto em companhias, como no próprio governo. 
Na literatura, é possível encontrar diversos trabalhos na área. Hussain et al. (2008) estudaram uma rede neural para prever a taxa de câmbio do dólar americano frente a outras três moedas. Coelho et al. (2008) investigam se os modelos obtidos a partir de redes neurais e o sistema nebuloso Takagi-Sugeno são capazes de prever a taxa de câmbio do real e o dólar americano, comparados, ainda, aos modelos autorregressivos. Além disso, Braga et al. (2010) propuseram um modelo NARMAX para medir a inflação. Por fim, trabalhos recentes demostram a alta confiabilidade em desenvolvimento de metodologias e estratégias para a identificação da inflação brasileira, como por exemplo, o trabalho de Silva and Marçal (2018). Contudo, no caso de sistemas multivariados, o alto número de regressores possibilita o desenvolvimento de novas abordagens para a redução de modelos e novas perspectivas de validação.

No entanto, nenhuma das técnicas apresentadas se preocupa com a validação e com o acerto de tendência dos respectivos modelos. Com isso, a proposta deste trabalho é desenvolver uma nova metodologia para a validação, IAT (Índice de Acerto de Tendência), pois a identificação da tendência para sistemas voláteis fornece novas perspectivas nos estudos de sistemas, como por exemplo, o reconhecimento da continuação de um movimento de alta ou de baixa, a reversão da tendência e, ainda, a redução do número de regressores, através do IAT, para modelos multivariados.

O artigo está dividido da seguinte forma: esta seção 1 descreve um breve panorama de identificação de sistemas e o estado da arte do trabalho. A seção 2 contém os conceitos necessários para o entendimento do restante do texto. Os métodos utilizados, bem como os resultados obtidos, estão indicados nas seções 3 e 4 , respectivamente. Por fim, a conclusão do trabalho e as suas perspectivas futuras estão contidas na seção 5 .

\section{CONCEITOS PRELIMINARES}

\subsection{Identificação de Sistemas}

A atividade de identificação de sistemas é fundamental na representação de estruturas, sendo muitas vezes empregada para melhorar o desempenho de sistemas. As técnicas de identificação de sistemas buscam representar o comportamento de um sistema real por meio de um modelo matemático, que deve reproduzir tanto as suas características estáticas quanto dinâmicas.

Para se identificar um sistema, é necessária a realização de 5 etapas, apresentadas em Ljung (1987) e mencionadas anteriormente. Visto isso, em problemas de modelagem de sistemas, é muito importante que o modelo final seja válido. Dessa forma várias técnicas foram desenvolvidas para a validação, como por exemplo, o uso de algumas propriedades dinâmicas, expoentes de Lyapunov e diagramas de bifurcação (Aguirre and Billings, 1995, 1994). Por fim, uma prática comum consiste em manipular uma massa de dados diferentes dos estimados, e aplicar os dados de entrada desses no modelo obtido. O resultado é comparado com os dados reais, e então é feita uma avaliação visual da capacidade do modelo em acompanhar novas entradas (Jacome, 1996).
Além disso, em identificação de sistemas, também são utilizados índices de validação baseados no erro do modelo. Entre os mais usados, estão o índice baseado na raiz quadrada do erro médio quadrático (RMSE, do inglês root mean square error) e o erro médio percentual absoluto (MAPE do inglês mean absolute percentage error). Entretanto, nem sempre é simples a obtenção de um modelo com erro baixo, pois modelos econômicos com índice RMSE baixo são, geralmente, muito complexos, além do processo de identificação ser trabalhoso.

\subsection{Modelos NARX polinomiais}

O modelo NARX polinomial (Nonlinear Auto-Regressive with eXogenous Inputs) abrange uma classe importante em sistemas não lineares de tempo discreto, que expressa o valor da saída $\mathrm{y}(\mathrm{k})$ em função de valores prévios dos sinais de entrada-saída, como mostra a Equação (1):

$$
\begin{aligned}
y(k)= & F^{\ell}\left[y(k-1), \ldots, y\left(k-n_{y}\right)\right. \\
& \left.u(k-d), \ldots, u\left(k-n_{u}\right)\right] \\
& +e(k)
\end{aligned}
$$

em que $n_{y}, n_{u}, d, u(k)$ e $y(k)$ são os atrasos em y, em $\mathrm{u}$, o tempo morto, o sinal de entrada e o sinal de saída, respectivamente e $e(k)$ representa ruído e possíveis incertezas. E ainda, a função $F^{\ell}$ representa uma função polinomial não linear do modelo com grau de não linearidade $l \in N$. Assim, a dinâmica do sistema pode ser reconstruída utilizando uma aproximação polinomial, em que $\ell$ é o grau máximo do polinômio utilizado para representar $F^{\ell}$ (Billings et al., 1988).

\section{METODOLOGIA}

\section{1 Índice de Acerto de Tendência}

Sistemas financeiros são sensíveis a vários tipos de interferências, dos mais diversos fatores, como crises econômicas, negociações envolvendo empresas, decisões políticas e econômicas, consumo da população, entre tantas outras variáveis. Dessa maneira, é difícil prever exatamente o comportamento do sistema.

No contexto de investimentos ativos, com maior risco, possuem maior possibilidade de altos retornos de investimento. Portanto, é conveniente a obtenção de um modelo capaz de antecipar o comportamento do ativo com um alto nível de acerto, aumentando a probabilidade de lucro por parte do investidor.

Dessa forma, para validar sistemas econômicos, foi proposto nesse trabalho o Índice de Acerto de Tendência (IAT) dado pela equação (2) que fornece um dado quantitativo em relação ao acerto da tendência do sistema pelo modelo.

$$
I A T=\left(1-\frac{1}{N-1} \sum_{k=1}^{N-1} \operatorname{sign}|\gamma(\mathrm{k})|\right) \times 100 \%
$$

em que $\gamma(k)=\operatorname{sign}(\Delta \hat{\mathrm{y}}(\mathrm{k}))-\operatorname{sign}(\Delta \mathrm{y}(\mathrm{k}))$ sendo $\Delta \hat{y}(k)=$ $\hat{y}(k)-\hat{y}(k-1)$ e $\Delta y(k)=y(k)-y(k-1)$ os valores obtidos do modelo e do sistema nos instantes $\mathrm{k}$ e k-1, 
respectivamente; $\operatorname{sign}($.$) é a função sinal e N$ o número de dados.

Pelo cálculo de $\operatorname{sign}(\Delta \hat{y})$ e $\operatorname{sign}(\Delta y)$ sabe-se que se a tendência foi de alta $(\operatorname{sign}()=1$.$) , baixa (\operatorname{sign}()=-1$. ou o valor manteve-se $(\operatorname{sign}()=0$.$) . Ao calcular-se \gamma(k)$ sempre que a tendência do modelo for igual a tendência do sistema $(\operatorname{sign}(\Delta \hat{y}(\mathrm{k}))=\operatorname{sign}(\Delta \mathrm{y}(\mathrm{k})))$ o resultado será nulo, do mesmo modo, quando a tendência for diferente $(\operatorname{sign}(\Delta \hat{\mathrm{y}}(\mathrm{k})) \neq \operatorname{sign}(\Delta \mathrm{y}(\mathrm{k})))$ o resultado será diferente de zero. Ao calcular a função sinal do valor absoluto de $\gamma(k)$, o valor será sempre zero quando as tendências forem iguais $(\operatorname{sign}|\gamma(\mathrm{k})|=0)$ e um valor positivo quando as tendências forem diferentes $(\operatorname{sign}|\gamma(\mathrm{k})|=1)$. Fazendo o somatório de $\operatorname{sign}|\gamma(\mathrm{k})| \forall \mathrm{k} \in \mathbb{N}: 1 \leq \mathrm{k} \leq \mathrm{N}-1$ e dividindo pelo número de vezes que a tendência foi calculada (N-1) temse a estatística de vezes que o modelo errou a tendência do sistema. Ao calcular o evento complementar $1-P\left(A^{c}\right)$ em que $P\left(A^{c}\right)$ é a estatística de vezes que o modelo errou do modelo errar, obtém-se o valor de IAT.

Com isso, o IAT nos dá a porcentagem de vezes em que o modelo acertou a tendência do sistema. Em modelos econômicos, o IAT, idealmente deve ser $100 \%$. Dessa forma, o economista pode afirmar qual será o comportamento do mercado financeiro no futuro, e determinar quais são as melhores medidas a serem tomadas.

\subsection{Identificação da inflação brasileira}

Como exemplo do uso da técnica proposta, foram obtidos modelos que buscam prever a inflação no mês seguinte, baseado-se na cotação do dólar e do índice Petróleo Brent.

O primeiro passo para a obtenção de um modelo que descreva a inflação brasileira, foi a escolha do índice de inflação. No Brasil, existem alguns indicadores de inflação, dentre os mais comuns estão o INPC (Índice Nacional de Preços ao Consumidor), o IGP-M (Índice Geral de Preços - Mercado) e o IPCA (Índice de Preços ao Consumidor Amplo). Para o presente trabalho, foram utilizados os dados do IPCA entre março de 2003 e agosto de $2018^{1}$, o qual é considerado o índice oficial de inflação do país.

A cotação do dólar foi escolhida, tendo em vista que o Brasil adotou o regime de taxa flutuante de câmbio, ou seja, a cotação do dólar e de outras moedas é determinada pelo mercado. Dessa forma, os preços dos insumos, industriais e comerciais, são voláteis e variam de acordo com a moeda estrangeira, influenciando diretamente na inflação do país (Scherer et al., 2010). Da mesma maneira, a cotação do Petróleo Brent foi escolhida, devido ao fato de que a indústria nacional é fortemente influenciada pelo preço do petróleo. Ademais, foram feitas as funções de correlação cruzada entre as entradas e notou-se uma correlação entre os dados.

Como representação matemática, foram utilizados os modelos polinomiais NARX devido à simplicidade na simulação de modelos e pela capacidade de modelar diversos comportamentos, lineares e não lineares. Vários trabalhos envolvendo previsão em sistemas financeiros utilizam esse tipo de representação matemática, como visto em

1 Dados disponíveis em: http://dados.gov.br/dataset/4448-indicede-precos-ao-consumidor-amplo-ipca-nao-comercializaveis.
(McHugh et al., 2018), em que se obteve um modelo NARX para previsão de preço de eletricidade, além de analisar a influência dos preços de combustíveis na composição do modelo.

A determinação e a quantidade dos regressores que vão compor o modelo são baseadas na taxa de redução de erro (ERR, do inglês error reduction ratio) (Korenberg et al., 1988), assim como no critério de informação de Akaike (AIC, do inglês Akaike information criterion) (Akaike, 1974). A taxa de redução de erro ranqueia os regressores baseada na sua contribuição para a redução do erro do modelo, enquanto o AIC indica o número de regressores que devem ser usados no modelo, de forma que ele não seja tão complexo.

$\mathrm{Na}$ etapa de estimação de parâmetros, foi utilizado o método dos mínimos quadrados ortogonais, dado pela equação abaixo:

$$
\theta=\left[\boldsymbol{\Psi}^{\mathbf{T}} \boldsymbol{\Psi}\right] \boldsymbol{\Psi}^{-\mathbf{1}} \mathbf{y}
$$

sendo que $\theta$ são os parâmetros do modelo, $\boldsymbol{\Psi}$ é a matriz de regressores e y é o vetor de saída do sistema. Dessa forma, são obtidos os parâmetros que minimizam o erro de predição do modelo.

Por fim, na etapa de validação, foram utilizados o IAT e o índice RMSE. Os resultados serão apresentados na seção seguinte.

\section{RESULTADOS}

A seguir serão apresentados os modelos NARX polinomiais obtidos para predição um passo a frente do índice IPCA para dois casos, para entrada única e saída única (SISO do inglês Single Input - Single Output); sendo um para a entrada Petróleo Brent, e outra para a cotação do dólar, e um caso em que foram utilizadas múltiplas entradas e saída única (MISO do inglês Multiple Input - Single Output) em que foram usados as duas entradas dos casos anteriores em conjuntos. Devido à alta complexidade do sistema financeiro, e à dificuldade de mensurar as inúmeras variáveis que o regem, um modelo que prevê a inflação de forma livre ou com mais passos à frente, se torna muito complexo; visto que uma previsão um passo à frente já é suficiente para muitas aplicações financeiras; como por exemplo, em investimentos no mercado de ações (Nenortaite and Simutis, 2005).

\subsection{Modelo SISO utilizando como entrada o índice mensal do dólar}

Seguindo as etapas de identificação de sistemas, obtiveramse os modelos para previsão da inflação. Para esse trabalho, foram utilizados 185 dados do IPCA, sendo que 155 dados foram destinados para a identificação, e mais 30 dados para a validação do modelo. Procurou-se, inicialmente, obter um modelo representativo utilizando apenas uma entrada, além de definir o máximo grau de não linearidade do sistema como sendo 2, com máximo atraso 4.

Utilizando, como entrada do modelo, o índice mensal do dólar fornecido pelo Banco Central do Brasil, obteve-se o 
modelo para o IPCA composto de 9 termos descrito pela equação 4,

$$
\begin{aligned}
\hat{y}(k)= & \theta_{1} y(k-1)+\theta_{2} u_{1}(k-1) \\
& +\theta_{3} y(k-2)+\theta_{4} y(k-4)^{2} \\
& +\theta_{5} y(k-4) y(k-3)+ \\
& +\theta_{6} y(k-3)^{2}+\theta_{7} u_{1}(k-1)^{2} \\
& +\theta_{8}+\theta_{9} u_{1}(k-1) y(k-1) .
\end{aligned}
$$

Os parâmetros $\theta_{i}$ estão descritos na Tabela 1 .

\begin{tabular}{|l|r|}
\hline Parâmetros & Valor \\
\hline$\theta_{1}$ & 1,1534 \\
\hline$\theta_{2}$ & $-4,0498$ \\
\hline$\theta_{3}$ & $-0,2278$ \\
\hline$\theta_{4}$ & 0,5147 \\
\hline$\theta_{5}$ & $-0,8342$ \\
\hline$\theta_{6}$ & 0,2667 \\
\hline$\theta_{7}$ & 3,9784 \\
\hline$\theta_{8}$ & 1,1805 \\
\hline$\theta_{9}$ & $-0,9453$ \\
\hline
\end{tabular}

Tabela 1. Parâmetros do modelo utilizando como entrada o índice mensal do dólar.

Calculando o IAT e o RMSE do modelo, foram obtidos os valores descritos na Tabela 2. A partir de tais valores da tabela, é possível perceber que o RMSE para os dados de validação é maior que 1 , o que significa que a média dos dados possui um erro menor que o modelo. Apesar disso, o modelo acertou a tendência em $75,86 \%$ das vezes, o que é satisfatório do ponto de vista econômico.

A figura 1 mostra o gráfico de tendência para o modelo da equação (4). Nas amostras onde há um $\otimes$, estão indicados os pontos onde a tendência dos dados coincide com a tendência do modelo.

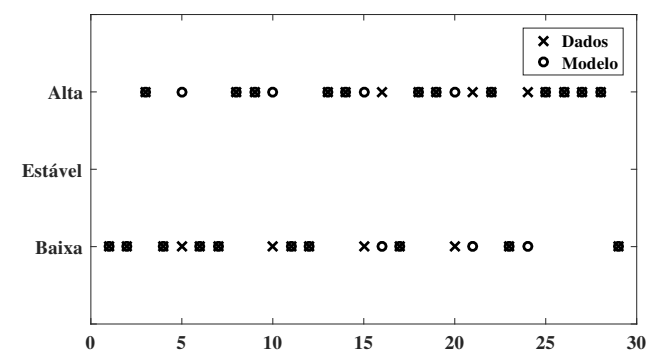

Figura 1. Gráfico de tendência para o modelo com a entrada sendo a cotação do dólar.

\begin{tabular}{|l|l|l|}
\hline & RMSE & IAT \\
\hline Identificação & 0,5717 & $81,33 \%$ \\
\hline Validação & 1,1666 & $75,86 \%$ \\
\hline
\end{tabular}

Tabela 2. Índices utilizados para validação do modelo utilizando como entrada o índice mensal do dólar.

4.2 Modelo SISO utilizando como entrada a cotação do Petróleo Brent

No segundo caso, para previsão da tendência do índice IPCA, foi utilizada como entrada a cotação do Petróleo
Brent. Seguindo as mesmas etapas de identificação, foi obtido um modelo composto de 10 regressores. O modelo está representado abaixo pela equação (5),

$$
\begin{aligned}
\hat{y}(k)= & \theta_{1} y(k-1)+\theta_{2} u_{1}(k-3) \\
& +\theta_{3} y(k-2) u_{1}(k-1) \\
& +\theta_{4} y(k-4)^{2} \\
& +\theta_{5} y(k-4) y(k-3) \\
& +\theta_{6} y(k-3)^{2} \\
& +\theta_{7} u_{1}(k-4) y(k-3) \\
& +\theta_{8} y(k-3) \\
& +\theta_{9} u_{1}(k-1)+\theta_{10} .
\end{aligned}
$$

Os parâmetros $\theta_{i}$ do modelo estão descritos na Tabela 3.

\begin{tabular}{|l|r|}
\hline Parâmetros & Valor \\
\hline$\theta_{1}$ & 0,6505 \\
\hline$\theta_{2}$ & 1,3236 \\
\hline$\theta_{3}$ & $-0,3376$ \\
\hline$\theta_{4}$ & 0,5416 \\
\hline$\theta_{5}$ & $-0,8053$ \\
\hline$\theta_{6}$ & 0,3678 \\
\hline$\theta_{7}$ & $-1,1394$ \\
\hline$\theta_{8}$ & 0,6166 \\
\hline$\theta_{9}$ & $-0,6845$ \\
\hline$\theta_{10}$ & $-0,1950$ \\
\hline
\end{tabular}

Tabela 3. Parâmetros do modelo utilizando como entrada o índice Petróleo Brent.

Calculando os índices de validação do modelo, obtiveramse os resultados expressos na Tabela 4. Baseado no IAT, foi possível notar que o modelo teve uma queda na qualidade, em relação ao representado na equação (4). Entretanto, através gráfico de tendência, representado na figura 2 notam-se pontos em que a equação (5) conseguiu acertar a tendência, mas o modelo da equação (4), não.

\begin{tabular}{|l|l|l|}
\hline & RMSE & IAT \\
\hline Identificação & 0,5792 & $80,67 \%$ \\
\hline Validação & 1,0264 & $58,62 \%$ \\
\hline
\end{tabular}

Tabela 4. Índices utilizados para validação do modelo utilizando como entrada o índice Petróleo Brent.

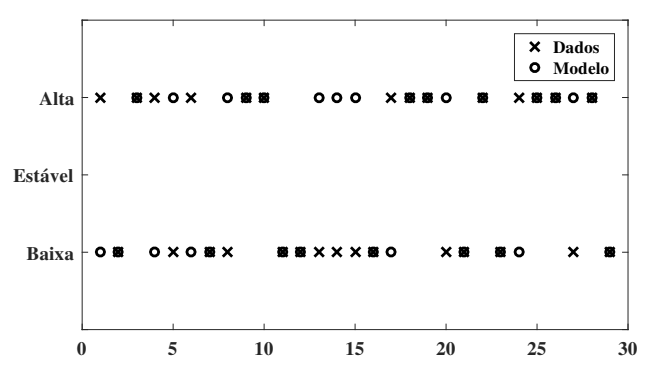

Figura 2. Gráfico de tendência para o modelo com a entrada sendo o índice Petróleo Brent. 


\subsection{Modelo MISO utilizando como entrada a cotação do dólar e Petróleo Brent.}

Com o objetivo de melhorar a qualidade de previsão do modelo, foi feita a modelagem utilizando como entradas a cotação do dólar e do Petróleo Brent concomitantemente. Seguindo os passos de identificação de sistemas, obteve-se um modelo composto de 8 regressores representado pela equação abaixo (Eq. (6)),

$$
\begin{aligned}
\hat{y}(k)= & \theta_{1} y(k-1)+\theta_{2} u_{2}(k-3) u_{1}(k-1) \\
& +\theta_{3} u_{2}(k-1) y(k-2) \\
& +\theta_{4} y(k-2) y(k-1) \\
& +\theta_{5} y(k-4)^{2}+\theta_{6} y(k-4) \\
& +\theta_{7} y(k-3)^{2}+\theta_{8} y(k-3) u_{2}(k-4) .
\end{aligned}
$$

Os parâmetros $\theta_{i}$ de tal modelo estão mostrados na Tabela 5 .

\begin{tabular}{|l|r|}
\hline Parâmetros & Valor \\
\hline$\theta_{1}$ & 0,5783 \\
\hline$\theta_{2}$ & 0,7689 \\
\hline$\theta_{3}$ & $-0,4460$ \\
\hline$\theta_{4}$ & 0,1625 \\
\hline$\theta_{5}$ & 0,4912 \\
\hline$\theta_{6}$ & $-0,7540$ \\
\hline$\theta_{7}$ & 0,3948 \\
\hline$\theta_{8}$ & $-0,2860$ \\
\hline
\end{tabular}

Tabela 5. Parâmetros do modelo utilizando como entradas o dólar e o índice Petróleo Brent.

Ao simular o modelo, foram encontrados os índices de validação descritos na Tabela 6 . Pode ser visto na tabela que o valor de IAT alcançou um valor satisfatório e diferente dos outros casos. O valor do RMSE se manteve próximo, tanto para os dados de identificação, quanto para os dados de validação. É possível notar que o modelo conseguiu descrever a tendência do sistema, conseguindo prever o comportamento do IPCA em $82,76 \%$ dos meses escolhidos para a validação do sistema. Tais afirmações são comprovadas com o gráfico de tendência da figura 3.

\begin{tabular}{|l|l|l|}
\hline & RMSE & IAT \\
\hline Identificação & 0,5682 & $84,00 \%$ \\
\hline Validação & 0,6070 & $82,76 \%$ \\
\hline
\end{tabular}

Tabela 6. Índices utilizados para validação do modelo utilizando como entradas dólar e índice Petróleo Brent.

\section{CONCLUSÃO}

Sistemas econômicos são influenciados por uma enorme quantidade de variáveis, e os modelos que representam fielmente com um pequeno erro entre os dados do sistema e a saída do modelo, são demasiadamente complexos. Porém, para algumas aplicações econômicas, prever o valor exato do sistema não é tão relevante quanto a obtenção de um modelo que acerte a tendência dos dados. Como aplicação do método, obtiveram-se três modelos que conseguem prever a inflação do país com bons níveis de

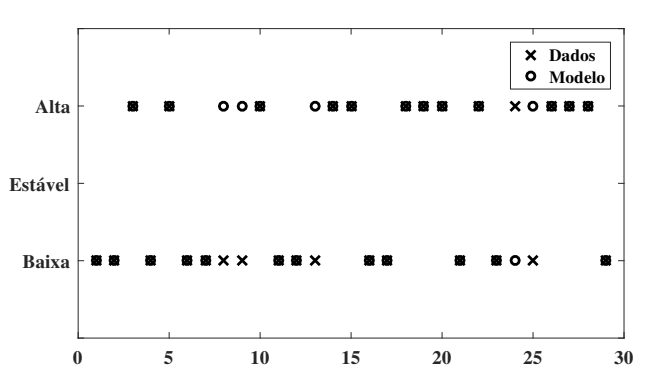

Figura 3. Gráfico de tendência para o modelo com a entrada sendo o dólar e o índice Petróleo Brent.

IAT. Especificamente, o modelo, vide equação 6 , foi o que mais se assemelhou ao sistema original, apresentando um $I A T=82,76 \%$.

$\mathrm{O}$ índice proposto, IAT, surge como uma alternativa para validar modelos, principalmente oriundos de sistemas econômicos, pois dependendo do índice quantitativo obtido pelo modelo, mais eficaz será a tomada de decisão de algum indivíduo e/ou equipe.

Como trabalho futuro, pretende-se utilizar o IAT como função custo, visando encontrar uma estrutura que seja apropriada para indicar a tendência do modelo.

\section{REFERÊNCIAS}

Aguirre, L.A. and Billings, S. (1994). Validating identified nonlinear models with chaotic dynamics. International Journal of Bifurcation and Chaos, 4(01), 109-125.

Aguirre, L.A. (2004). Introdução à identificação de sistemas: técnicas lineares e nẫo-lineares aplicadas a sistemas reais. UFMG, Belo Horizonte.

Aguirre, L.A. and Billings, S. (1995). Retrieving dynamical invariants from chaotic data using narmax models. International Journal of Bifurcation and Chaos, 5(02), 449-474.

Akaike, H. (1974). A new look at the statistical model identification. IEEE transactions on automatic control, 19(6), 716-723.

Billings, S., Korenberg, M., and Chen, S. (1988). Identification of non-linear output-affine systems using an orthogonal least-squares algorithm. International Journal of Systems Science, 19(8), 1559-1568.

Braga, D.C.d.S., Nepomuceno, E.G., and Cerqueira, S.A.A.G. (2010). Aplicação de algoritmos genéticos na determinação de estruturas de um modelo polinomial NARMAX. Anais do Nono Simpósio de Mecânica Computacional.

Buonomo, B., Carbone, G., and d'Onofrio, A. (2018). Effect of seasonality on the dynamics of an imitationbased vaccination model with public health intervention. Mathematical Biosciences \& Engineering, 15(1), 299321.

Coelho, L.S., Santos, A.P., and da Costa Jr, N.A. (2008). Podemos prever a taxa de câmbio brasileira? Evidência empírica utilizando inteligência computacional e modelos econométricos. Gestão $\&$ Produção, 15, 635-647.

De Nicolao, G. (1997). System identification: Problems and perspectives. In 12th Workshop on Qualitative Reasoning, volume 3, 1-2.

Hussain, A.J., Knowles, A., Lisboa, P.J., and El-Deredy, W. (2008). Financial time series prediction using poly- 
nomial pipelined neural networks. Expert Systems with Applications, 35(3), 1186-1199.

Jacome, C.R.F. (1996). Uso de conhecimento prévio na identificação de modelos polinomiais NARMAX. Tese de Mestrado, Universidade Federal de Minas Gerais, Belo Horizonte.

Korenberg, M., Billings, S., Liu, Y., and McIlroy, P. (1988). Orthogonal parameter estimation algorithm for non-linear stochastic systems. International Journal of Control, 48(1), 193-210.

Ljung, L. (1987). System identification: theory for the user. Prentice-hall.

McHugh, C., Coleman, S., Kerr, D., and McGlynn, D. (2018). A linear polynomial narmax model with multiple factors to forecast day-ahead electricity prices. In 2018 IEEE Symposium Series on Computational Intelligence (SSCI), 2125-2130. doi:10.1109/SSCI.2018.8628694.

Nenortaite, J. and Simutis, R. (2005). Adapting particle swarm optimization to stock markets. In 5th International Conference on Intelligent Systems Design and Applications (ISDA'05), 520-525. IEEE.

Nguyen, Q.H. (2019). Growth model with financial deepening and productivity heterogeneity. The Japanese Economic Review, 70(1), 123-140.

Scherer, F.L., Kowaleski, P.S., and Rezende Júnior, S.V.d. (2010). A dinâmica da inflação brasileira após o Plano Real. Vitrine da Conjuntura Econômica, Curitiba, 3(6).

Silva, A.M. and Marçal, E.F. (2018). Descobrindo modelos de previsão para a inflação brasileira: Uma análise a partir de uma gama ampla de indicadores. Estudos Econômicos (São Paulo), 48(3), 423-450.

Waseem, M. and Sadiq, M.U. (2018). Application of model-based systems engineering in small satellite conceptual design-a sysml approach. IEEE Aerospace and Electronic Systems Magazine, 33(4), 24-34. 